\title{
Gestión del urbanismo y administración urbana: Los pies de barro en el despliegue territorial del neoliberalismo en Chile
}

Héctor Guillermo Gaete Feres

\section{Filiación}

Académico Universidad del Bío - Bío.

\section{Resumen}

En Chile, el abordaje eficiente de los problemas urbanos requiere enfrentar un asunto central pero rezagado de los procesos de modernización impulsados en el marco del modelo económico neoliberal vigente: la ineficiencia en la administración y gestión del desarrollo territorial. Ambas constituyen lastres que afectan el desempeño del sistema económico y el logro de los objetivos del país en calidad de vida para las personas. El artículo busca relevar, revisar el estado del arte y proponer líneas de trabajo hacia soluciones.

\section{Palabras Claves}

Administración, gestión, desarrollo territorial, neoliberalismo.

\begin{abstract}
Management of urbanism and urban administration. The Mud Feet in the Territorial Deployment of the Neoliberalism in Chile. In Chile, the efficient boarding of the urban problems requires to face a central but left behind subject of the impelled processes of modernization in the frame of the effective neoliberal economic model: inefficiency in the administration and management of the territorial development. Both constitute ballasts that affect the performance of the economic system and the profit of the objectives of the country in quality of life for the people. I articulate looks for to release, to review the state-of-the-art and to propose lines of work towards solutions.
\end{abstract}

\section{Key words}

Administration, management, territorial development, neoliberalism.

\section{Sumario}

Introducción

1. En Chile: Mercado Abierto y Estado Replegado (¿Ausente?).

2. Gestión del Urbanismo y Administración Urbana. Una Modernización Pendiente.

3. Estado del arte: Los aportes fundamentales y los desafíos.

4. Los Esfuerzos más recientes en Chile.

5. Modernización de la Administración y Gestión Urbanas.

6. El Caso de Singapur: rediseñar la administración para ganar competitividad.

7. El Caso de Curitiba: liderazgo y asociatividad.

8. Conclusiones y proposiciones

Referencias 


\section{I ntroducción}

El crecimiento económico y el desarrollo de Chile, por su fuerte tasa de concentración poblacional y productiva en áreas urbanas, dependen fuertemente de lo que ocurra con el desempeño de sus ciudades y de los sistemas que allí se despliegan. Es decir, el país está frente a la necesidad de que las áreas urbanas deban operar bien, para así poder continuar con el desempeño eficiente de su economía a escala macroeconómica y para avanzar hacia mejores resultados a nivel microeconómico que se traduzcan en un mayor bienestar y calidad de vida para las personas.

Dada la importancia estratégica de las ciudades, abordar adecuadamente los problemas urbanos es un tema central y, para hacerlo con eficiencia en el Chile de hoy, se requiere enfrentar dos asuntos centrales que han quedado rezagados o fuera de los llamados procesos de modernización llevados adelante en el marco del modelo económico neoliberal vigente. De una parte, la ineficiencia de la administración y gestión del desarrollo territorial $y$, de otra, la insuficiencia de su financiamiento. Ambos asuntos prácticamente no han sido abordados y constituyen lastres relevantes.

Como la gestión del urbanismo y la administración urbana forman parte, en Chile y en la mayor parte del mundo, de la esfera de acción de la Administración Pública, constituyen herramientas del Estado para asegurar el bien común en el territorio; por lo que resultan ser claves para la orientación y regulación del desarrollo integral en las ciudades.

Lo anterior es crucial porque la conducción adecuada de los asentamientos humanos se sostiene en buena parte "por y en" su staff administrativo. Por ello es que visualizamos la modernización de la administración pública vinculada al ámbito urbano como un elemento central para el desarrollo de la ciudad.

En ese contexto, no resultan ser menores los asuntos relacionados con el diseño de modelos de gestión y administración urbanas, la articulación entre los sectores público y privado/comunitario ${ }^{1}$, la velocidad de respuesta a los usuarios del sistema por parte de los órganos gubernamentales, la capacidad técnica de los mismos, los procedimientos, métodos e instrumental para la administración y gestión, la orientación al servicio de las necesidades de los habitantes, de la actividad económica y de la producción.

Lo planteado es necesario visualizarlo, por cierto, en términos apropiados al entorno, o sea, en el marco y al alcance de la realidad del Chile actual perfilado por el modelo económico, las condiciones sociales y políticas, y las desigualdades regionales actualmente vigentes.

Creemos, además, que resulta más provecho para el país trabajar la modernización pendiente del soporte administrativo y gestor del urbanismo, en el contexto de escasez de recursos humanos, de equipamiento y financieros, propio de las regiones, derivado de la acentuada macrocefalia que presenta el sistema urbano chileno, encabezado por su

\footnotetext{
${ }^{1}$ Usamos la dupla privado/comunitario para incluir no sólo al tradicional sector privado sino también a la vasta y rica organización social subyacente en el país que incluye: Corporaciones, Juntas de Vecinos, etc. 
metrópolis capital y su área de atracción inmediata. Ello por lo determinante que esta realidad es en el despliegue cotidiano de la administración y gestión del urbanismo.

En el presente artículo pretendemos poner de relieve los aspectos indicados, dar cuenta del avance y del rezago del conocimiento, revisar casos paradigmáticos y proponer líneas de trabajo hacia posibles soluciones.

\section{En Chile: Mercado abierto y Estado replegado (¿ausente?)}

Chile hoy se destaca, y se le reconoce en el ámbito mundial, por ser una de las economías más abiertas del planeta y por exhibir altas tasas de crecimiento económico ${ }^{2}$. Esta economía se rige por un modelo caracterizado por el libre juego de bienes y servicios en un marco en que los factores de producción operan también con amplia libertad teniendo al mercado como su principal asignador de recursos (también se le ha denominado: modelo neoliberal, ortodoxo o monetarista, el mayor ejemplo de ortodoxia de los últimos treinta años, etc. ).

La economía chilena abierta, vigente ya desde hace unos treinta años, no se responsabiliza o no se hace cargo de los efectos indeseables que genera, es decir, no internaliza las deseconomías y opera en un contexto caracterizado, a su vez, por recién iniciados o inexistentes mecanismos que mitiguen los impactos negativos al desplegarse el modelo económico en el proceso cotidiano de producción de bienes y servicios.

Así, por ejemplo, el factor mano de obra se incorpora al modelo con máxima flexibilidad en la contratación/despido; pero con recientes mecanismos de protección a las personas (seguro de desempleo) ${ }^{3}$; máxima flexibilidad en el uso de los recursos físicos (tierra, mar, aire, agua); pero con recientes y mínimos sistemas de evaluación/monitoreo de impacto ambiental; expeditos mecanismos de acceso al crédito inmobiliario (Lo que posibilitó una gran expansión de la producción de unidades de viviendas); pero sin la adopción de estándares adecuados, por ejemplo los de la Organización Mundial de la Salud de Naciones Unidas, OMS, con relación a calidad, superficie, urbanización; áreas verdes, equipamiento educacional, comunitario, social y de seguridad.

Del mismo modo la implantación del modelo económico neoliberal, a partir del año 1973 bajo la administración militar, trajo consigo - por razones ciertas de eficiencia y de exigencias de reducción del gasto público; pero también ideológicas - una disminución drástica del tamaño del Estado medido este en cantidad de funcionarios (aunque no necesariamente de funciones) y en la privatización de sectores de la economía especialmente en Telecomunicaciones y Energía. Todo ello en modalidad análoga al despliegue de la economía con relación a sus efectos descrito anteriormente, es decir, sin hacerse cargo de los impactos negativos; en este caso, sobre la capacidad de administración y gestión necesarias, o sea, sin arbitrar los sistemas y mecanismos para fortalecer la eficiencia del Estado en su responsabilidad de cautelar y promover el bien

\footnotetext{
$27,0 \%$ promedio en los $90 ; 5,4 \%$ el $2000 ;$ y 2,4\% proyectado al 2001 . El mayor crecimiento en Latinoamérica y uno de los cinco países con mayor crecimiento en el mundo. Véase Ffrench-Davis, 2001.

${ }^{3}$ La nueva ley de Laboral se aprobó recién en agosto 2001 y ha sido fuertemente criticada desde los sectores empresariales y de trabajadores.
}

Revista de Urbanismo $N^{\circ} 7$ - Enero de 2003 
común tal y como lo establece la Constitución Política aprobada en el mismo gobierno militar y que se mantiene aún vigente.

Es así como hoy Chile se caracteriza, además de por su economía abierta con grandes éxitos macroeconómicos medidos en altas tasas de crecimiento promedio anual, por un muy negativo desempeño de su microeconomía ${ }^{4}$. Por ejemplo: fuerte concentración de la riqueza (la segunda peor distribución en el ámbito mundial); gran cantidad de horas de trabajo per cápita (el primer país en el ámbito mundial) con baja productividad de la mano de obra (de los más bajos en el ámbito mundial); grandes impactos medioambientales ( $62 \%$ del territorio en proceso de desertificación); fuerte concentración poblacional y de la producción acompañados de alto centralismo en las decisiones (la Región Metropolitana -RM- de Santiago, capital nacional, concentra el $40 \%$ de la población en el $2 \%$ de territorio; el $48 \%$ del producto interno bruto; el $55 \%$ de la inversión pública y el $90 \%$ de la privada son decididas en la capital; 46 de los 47 grupos económicos tienen su sede en la RM; $97 \%$ de la operación del mercado de valores; $80 \%$ del Gasto Público en Cultura; $69 \%$ de los fondos para investigación científica; etc. ${ }^{5}$ ); grandes problemas de coordinación entre distintos niveles funcionales y territoriales de la acción del Estado evidenciados en permanentes conflictos urbanos y de competencia jurisdiccional entre instituciones; colisiones persistentes entre los distintos niveles de gestión gubernamental para la aplicación de las políticas públicas; fuertes deficiencias del aparato del Estado en la promoción, administración, gestión y control del_ordenamiento territorial y desarrollo urbano con su impacto negativo en la búsqueda de soluciones a los problemas de las ciudades, en la promoción del desarrollo urbano y en la evaluación y seguimiento de las políticas, planes, programas y proyectos en pos de alcanzar mayor eficiencia en el desempeño de las ciudades acompañada de adecuados niveles de calidad de vida para las personas.

Por cierto, estos problemas se traducen, en la gestión cotidiana, a una baja velocidad de respuesta al sector privado/comunitario en los procesos de evaluación y aprobación de proyectos, en la entrega de permisos de construcción, en los procesos de inspección y control de obras, en viabilizar el financiamiento para el desarrollo urbano, en rentabilizar socialmente la ciudad, en asegurar condiciones adecuadas de habitabilidad para todos los ciudadanos, etc.

Una primera y adelantada conclusión es que resulta altamente urgente introducir análisis e incorporar conocimiento con miras a contribuir al abordaje y solución de un problema central que enfrenta hoy el urbanismo en Chile: la baja velocidad de respuesta de los órganos gubernamentales a cargo de la administración y gestión urbana, en el marco del modelo económico neoliberal y de la realidad social y política actualmente vigentes.

Chile en el territorio se estructura y organiza en torno a cuatro grandes áreas de concentración poblacional: el Gran Santiago, ciudad capital; el Gran Valparaíso; el Gran Concepción; y la Conurbación Coquimbo - La Serena; las que juntas representan tres cuartas partes de la población nacional ${ }^{6}$. Sin embargo, en la región capital se concentran

\footnotetext{
${ }^{4}$ Para una exhaustiva revisión del experimento neoliberal en Chile véase: Ffrench-Davis, 2001, pp.26-29.

${ }^{5}$ CONAREDE, Consejo Nacional para la Regionalización y el Desarrollo, 1998. El CONAREDE es una Comisión Presidencial multisectorial de la que el autor del presente artículo es miembro.

${ }^{6}$ MINVU 1, 2001, sobre datos del INE, Instituto Nacional de Estadísticas. 
fuertemente la población, la producción, los recursos humanos calificados; financieros, de equipamiento, tecnológicos y decisionales, lo que produce una relación de escala fuertemente diferencial con las otras áreas caracterizadas como de tamaño medio.

Por lo mismo y en el marco de lo anterior, una segunda idea es que resulta más provecho para el país trabajar el tema en un contexto de escasez de los recursos indicados lo que implica, dada la fuerte macrocefalia que presenta el sistema urbano chileno, concentrar esfuerzos fuera de la Capital y de su área de atracción inmediata por lo determinante que es en el despliegue cotidiano de la administración y gestión del urbanismo. Esto, por cierto, nos exige articular fuertemente las políticas públicas tendientes a la Descentralización y Regionalización con las que buscan fortalecer las capacidades técnicas de las regiones en materia de Administración y Gestión Urbanas.

\section{Gestión del Urbanismo y administración urbana. Una modernización pendiente}

Chile por lo anteriormente indicado y por su fuerte tasa de concentración poblacional y productiva en áreas urbanas ( 180 ciudades y pueblos concentran el $86 \%$ de la población, y el $86 \%$ del producto interno bruto ${ }^{7}$ ), tiene sobre si la necesidad de que para continuar con el desempeño eficiente de su economía a escala macroeconómica y para avanzar hacia mejores resultados a nivel microeconómico que se traduzcan en un mayor bienestar para las personas, depende fuertemente de lo que ocurra, a su vez, con el desempeño en las ciudades y de los sistemas que allí se despliegan.

Las características que presenta el desarrollo urbano de las ciudades chilenas dejan ver que este es más bien el resultado no deseado (o el mal necesario) de la actividad económica. Los problemas de crecimiento espontaneo y los consiguientes efectos sobre las áreas agrícolas, el ineficiente uso del suelo derivado del crecimiento en extensión con densidades extraordinariamente bajas y el incremento notable del costo de transporte en el ingreso familiar, constituyen impactos indeseables sobre la idea declarada de asegurar un desarrollo urbano más equitativo y amable para las personas.

Por otra parte los intentos por reaccionar se ven enfrentados a la escasa capacidad técnica de los órganos responsables del desarrollo urbano y a la insuficiencia de método e instrumental para abordar con mayor racionalidad la acción para influir en un desarrollo que satisfaga las necesidades de los habitantes y de la propia actividad económica y de producción.

Se constituye en una necesidad urgente el desarrollo del conocimiento en mecanismos e instrumentos de gestión que estén al alcance de la aplicación acorde a la realidad de Chile, con sus desigualdades regionales derivadas de la distribución de los recursos humanos y capacidad técnica.

Los recursos orientados por el Estado hacia la consecución de un desarrollo urbano más equilibrado se ven sistemáticamente enfrentados, sobre todo en las ciudades intermedias, a la escasa capacidad técnica para formular e implementar programas y proyectos que integren a la población a los beneficios del desarrollo económico y, por otra parte, la

\footnotetext{
${ }^{7}$ MINVU 2, 2001

Revista de Urbanismo $N^{\circ} 7$ - Enero de 2003
} 
insuficiencia de dichos recursos han puesto en evidencia tanto la necesidad de incorporación del sector privado/comunitario en alianza estratégica con el Estado como la urgencia por implementar modalidades para incrementar la cuantía de los recursos.

A medida que los países sufren la agudización de las rigideces y restricciones al crecimiento económico, resulta necesario acentuar el cuidado por la sintonía fina del despliegue del modelo y se hace evidente que las características que el estilo de desarrollo urbano adopte se tornan cada vez más significativas para el cumplimiento de los objetivos y metas del desarrollo económico y social.

En ese contexto, el diseño de modelos de gestión urbana acordes a los desafíos de integración de la población a los beneficios del desarrollo y a la articulación de los sectores público y privado/comunitario se constituyen en una necesidad crucial para el desarrollo de Chile.

A partir de ello se hace necesario crear y recrear modalidades de gestión urbana que permitan asumir los desafíos del país y el problema central es cómo se la satisface en sintonía con las características propias del estado actual de Chile, haciendo un parangón con lo que podríamos denominar tecnologías apropiadas. Es decir, cómo desarrollar modelos de administración y gestión urbana que sean apropiados y que puedan por lo tanto tener mayor factibilidad de ser asumidos e implementados acorde a la realidad del aparato público y privado/comunitario, de los recursos disponibles y de los escenarios que generan el marco jurídico y administrativo.

En este contexto deben asumirse las desigualdades regionales existentes hoy en Chile y que se derivan de la concentración de la población y de los recursos claves para la gestión. La formulación de políticas homogéneas para Chile se han traducido en fracasos que van desde la no-implementación de las mismas hasta la falta de capacidad técnica para hacer uso de los recursos asignados al presupuesto anual en distintas regiones.

El abordaje eficiente de los problemas urbanos en el Chile de hoy requiere, por lo tanto, enfrentar dos asuntos centrales que han quedado rezagados o fuera de los llamados procesos de modernización llevados adelante en el marco del modelo económico neoliberal vigente: de una parte, la ineficiencia en la administración y gestión del desarrollo territorial y, de otra, la insuficiencia de financiamiento para el mismo. Ambos asuntos prácticamente no han sido abordados ${ }^{8}$ y constituyen lastres relevantes del modelo económico neoliberal actualmente imperante.

Para el caso de la problemática del financiamiento existe ya una interesante acumulación de esfuerzos investigativos que se han traducido, a su vez, en un importante consenso (aunque aún sólo académico, científico y técnico; pero no político) en la dirección de indexar las soluciones a procesos y mecanismos de recuperación de plusvalías generadas

\footnotetext{
${ }^{8}$ La Política Urbana de mayor duración en Chile fue diseñada en 1985 en el Gobierno del General Pinochet (véase MINVU, 1985). Fue derogada en el Gobierno de Lagos, por decreto $N^{\circ} 259$, sólo en octubre de 2000 y aún no cuenta con reemplazo oficial, aunque el 25 de junio 2001 el Presidente dio el inicio a los estudios y actividades para la Reforma Urbana (véase LAGOS 2, 2001). Los esfuerzos vinculados al financiamiento se han orientado a la vivienda, especialmente a la implementación de mecanismos de crédito bancario para los sectores de ingresos medios - altos y al sistema de subsidios para los de ingresos bajos. 
en el suelo privado por las decisiones públicas (regulación e inversión, principalmente) ${ }^{9}$. Sin embargo en el ámbito de la administración y gestión urbanas prácticamente no hay estudios que vayan más allá de la declaración de principios en torno a la necesidad de ser eficientes y eficaces; pero que no se acercan al análisis de la problemática ni, por cierto, a la solucionática. He incluso en la propia línea de investigación de los mecanismos de financiamiento aún no se aborda el asunto central: los recursos institucionales, administrativos y de gestión necesarias para realizar una efectiva recuperación de las plusvalías que permita avanzar en el financiamiento del desarrollo urbano.

Al respecto estimamos que, aunque con sus complejidades propias que en primera instancia lo muestran como un asunto centrado en si mismo, el financiamiento forma parte del contexto mayor que implica la administración y gestión del urbanismo y sólo es posible separarlo para fines de análisis.

Es decir, tanto para el caso específico del financiamiento vía recuperación de plusvalías como para el desempeño general de la economía en Chile y el rol que cumplen las ciudades y sistemas urbanos, se tiende a responder al qué hacer; con grados menores de avance en el cómo hacerlo; pero no se responde a las preguntas del cómo organizarlo; cómo implementarlo; con quién hacerlo; cuándo hacerlo; con qué tiempos; a qué costo y; con qué financiamiento.

Resulta necesario entonces aportar al conocimiento por la vía de abordar estás ultimas preguntas claves y proponer el o los elementos que permitan aproximarse a modelos de administración y gestión del urbanismo ajustados a la realidad económica, cultural, social, tecnológica y política chilena, en el contexto regional que brinda la realidad concreta de las ciudades intermedias en las regiones de Chile y en el marco de los grandes objetivos de regionalización y descentralización actualmente vigentes.

\section{Estado del arte: los aportes fundamentales y los desafíos}

Tal como lo indican Castells y Borja: “El desarrollo urbano está íntimamente ligado a la evolución socio-económica, política y cultural de una región", por lo cual cada una de ellas presenta características propias en el proceso de urbanización como es el caso de Latinoamérica. (Castells y Borja; 1988; pp.9). Estos autores identifican la crisis urbana latinoamericana como producto del modelo económico dependiente imperante y que, a medida que se profundiza, amplifica la crisis económica “... poniendo de relieve el desface creciente entre los problemas cotidianos (...) y los instrumentos técnicos e institucionales con que contamos para su tratamiento. Sin embargo, en aquellas situaciones en donde una renovación política ha permitido poner la Administración Pública al servicio del ciudadano y en donde una gestión rigurosa e imaginativa ha empezado a llevarse a la práctica, las condiciones de vida del pueblo han mejorado y la tendencia al deterioro comienza a frenarse, permitiendo alumbrar la esperanza de un futuro mejor para nuestras ciudades". (Castells y Borja; 1988; pp.17). Ello implica que, a pesar de la preeminencia de un modelo económico hegemónico, que predefine un rol dependiente a nuestros países, a la administración y gestión del urbanismo se les asigna un lugar central en la dirección de alcanzar niveles crecientes de calidad de vida. El planeamiento

\footnotetext{
${ }^{9}$ Para una completa revisión del tema Financiamiento Urbano en la región latinoamericana véase en bibliografía: SMOLKA y FURTADO Editores, Recuperación de Plusvalías en América Latina. 
urbano es “... en primer lugar un proceso político, de conflicto, representación de intereses y negociación institucional (...). De ahí que la condición previa para un tratamiento técnico eficaz de la crisis urbana sea la existencia de condiciones políticoadministrativas que relacionen dinámicamente la sociedad, el Estado y la gestión urbana. El municipio juega un papel clave en esta trama de relaciones. (Castells y Borja; 1988; pp.17) Es este planteamiento el que a pesar de su estructuralismo abre un esperanzador intersticio por el cual avanzar hacia mejorar la calidad de vida urbana mediante modelos y mecanismos adecuados para la administración y gestión urbana en nuestras ciudades.

La visualización de los asuntos de futuro vinculados a la administración y gestión urbana en el contexto latinoamericano se insinuaban ya en 1989 por la Comisión Económica para América Latina (CEPAL) de la Organización de Naciones Unidas (ONU) al indicar, respecto de la crisis urbana en la región, que: “...al conjunto de fenómenos a que se da el calificativo de críticos no son pasajeros, y que, más bien, son una manifestación del agotamiento del sistema y, por lo mismo, indicativos de cambios estructurales..."; y agrega que: “... esto significa que, al menos en lo que se refiere al ordenamiento territorial, a la planificación y gestión de los asentamientos humanos y al control de la calidad del hábitat, se presenta el ineludible desafío de buscar formas de acción alternativas que permitan apartarse de conceptos y esquemas originados en contextos económicos, sociales y culturales diferentes a los que prevalecerán en el futuro. (Cepal, 1989; pp.12-13)". Para ello se requerirá una fuerte convergencia de imaginación, racionalidad, voluntad política, capacidades técnicas, innovación conceptual, y “... mayor preparación institucional y de personal para aprovechar los recursos naturales, capacidades humanas y características culturales hoy insuficientemente utilizadas. La reestructuración de las políticas de asentamiento, la revisión del aparato institucional que apoya la gestión de los asentamientos humanos y la capacitación de los actores que intervienen en el proceso se constituyen, en este contexto, en una empresa ineludible que exige profundos cambios conceptuales e institucionales." (Cepal, pp13; op.cit). Sin embargo el organismo internacional no se aproxima a la propuesta de modelos de organización ni a mecanismos de administración o financiamiento.

En la misma dirección, la ONU en su declaración de Estambul para los Asentamientos Humanos (O.N.U.; 1996), indica que los gobiernos deben promover el fortalecimiento de los mecanismos de administración y gestión urbana con miras a elevar la calidad de vida la población, prestando especial atención a los aspectos relacionados con la descentralización y fortalecimiento de las capacidades locales para la conducción de dichos asentamientos. De hecho, en el Plan de Acción Mundial definido en la Conferencia HABITAT II, se indica que: La administración pública municipal puede contribuir eficazmente a que los asentamientos humanos sean viables, equitativos y sostenibles, puesto que ese nivel de administración es el más próximo al ciudadano. Es necesario que los gobiernos reconozcan la función esencial de las autoridades locales en lo que respecta a proporcionar servicios y poner al ciudadano en condiciones de lograr el desarrollo económico, el bienestar social y la protección medioambiental de sus comunidades locales, así como en lo concerniente a la cooperación internacional entre autoridades locales. Las autoridades locales pueden construir, explotar y mantener la infraestructura económica, social y medioambiental, supervisar los procesos de planificación, fijar políticas ecológicas locales y prestar asistencia en la aplicación de políticas ecológicas nacionales y subnacionales. Desempeñan un papel vital en lo que respecta a educar y 
Gestión del urbanismo y administración urbana: Los pies de barro en el despliegue territorial del neoliberalismo en Chile

movilizar a los ciudadanos y responder a la demanda pública para promover el desarrollo sostenible. ${ }^{10}$

Específicamente la ONU, a través de su Plan de Acción Mundial recomienda medidas a los países miembros en la idea de: “... asegurar la descentralización y el fortalecimiento de las autoridades locales y sus asociaciones y redes, los gobiernos a los niveles apropiados deben" ${ }^{11}$ :

a) examinar y adoptar, según proceda, políticas y marcos jurídicos de otros Estados que aplican con eficacia la descentralización;

b) examinar y revisar, según proceda, la legislación a fin de aumentar la autonomía local en la toma de decisiones, la aplicación, la movilización y utilización de recursos, especialmente en lo que respecta a los recursos humanos, técnicos y financieros y el desarrollo de empresas locales, en el marco general de las estrategias nacionales, sociales, económicas y ambientales, así como alentar la participación de los habitantes en la adopción de decisiones en lo relativo a sus ciudades, vecindarios o viviendas;

c) desarrollar la educación cívica para poner de relieve la función de las personas en sus respectivas comunidades;

d) prestar apoyo a las autoridades locales mediante la revisión de los sistemas de generación de ingresos fiscales;

e) fortalecer, en caso necesario, la capacidad de las instituciones de educación, investigación y formación para proporcionar formación continua a funcionarios electos, gerentes y profesionales en cuestiones relacionadas con el urbanismo, por ejemplo, planificación, técnicas de gestión de tierras y recursos y finanzas municipales;

f) facilitar el intercambio de tecnología, experiencia y conocimientos prácticos en materia de gestión, vertical y horizontalmente, entre el Gobierno y las autoridades locales en lo que se refiere a suministrar servicios, controlar los gastos, movilizar recursos, fomentar las asociaciones y desarrollar empresas locales, entre otras cosas mediante programas de asociación técnica y de intercambio de experiencias;

g) destacar los logros de las autoridades locales reuniendo datos desglosados por sexo, edad e ingresos y analizando comparativamente y difundiendo información sobre las prácticas innovadoras en cuanto al suministro, funcionamiento y mantenimiento de bienes y servicios públicos, a la atención de las necesidades de sus ciudadanos y al aprovechamiento del potencial fiscal y de otra índole de sus ciudades;

h) alentar la institucionalización de una amplia participación, incluidos mecanismos de consulta, en la adopción de decisiones y los procesos de gestión a nivel local;

i) reforzar la capacidad de las autoridades locales para recabar la participación de los sectores privado y comunitario en la fijación de objetivos y de prioridades locales y normas ecológicamente racionales para el desarrollo de infraestructura y el suministro de servicios y para el desarrollo económico local;

j) fomentar el diálogo entre todos los niveles de la administración y los sectores privado y comunitario y otros representantes de la sociedad civil, a fin de mejorar la planificación y la coordinación;

\footnotetext{
10 N.U./HABITAT II, PROGRAMA, Cap. IV, Plan de Acción Mundial: Estrategias para la Aplicación, Letra C, párrafo 102, Estambul, junio 1996.

${ }^{11}$ Op. Cit., Cap. IV, Letra D, párrafo 180, PROGRAMA, Cap. IV, letras indicadas.

Revista de Urbanismo N7 - Enero de 2003 
k) en el marco de la administración, establecer asociaciones entre el sector público y los ciudadanos privados con miras a la innovación urbana, y analizar, evaluar y difundir información sobre las asociaciones que hayan tenido éxito;

I) recoger, analizar y divulgar, según proceda, información comparativa, desglosada por sexo, edad e ingresos, sobre las realizaciones de las autoridades locales en lo tocante a satisfacer las necesidades de sus ciudadanos;

m) reforzar las medidas tomadas para erradicar la corrupción y garantizar una mayor transparencia, eficiencia y participación comunitaria en la gestión de los recursos locales;

n) facilitar a las autoridades locales y sus asociaciones o redes la adopción de iniciativas en la esfera de la cooperación nacional e internacional, en particular para compartir información sobre las prácticas adecuadas y los enfoques innovadores de la gestión sostenible de los asentamientos humanos;

o) reforzar la capacidad de los gobiernos centrales y de los locales mediante cursos de capacitación en hacienda y gestión urbanas, destinados a funcionarios públicos electos y gestores."

Es decir la organización multinacional establece con alta prioridad, la necesidad de incrementar la eficiencia de la administración y gestión locales vinculadas a: descentralización del poder de decisión, incremento de los niveles de capacitación de los funcionarios y técnicos, innovaciones en los mecanismos de financiamiento, incremento de la asociatividad y cooperación entre ciudades y países, revisión de las legislaciones con miras a limpiarlas de burocracia, mejoramiento de los mecanismos de información, incorporación del sector privado/comunitario, etc.

Lo anterior implica que la modernización de la Administración Pública se percibe como un elemento central para el desarrollo de la ciudad y ello porque, tal como indica Fernández Güell, “... la operativa ordenada y sistemática de las funciones urbanas depende en gran medida del aparato administrativo que la sustenta" (Fernández, 1997; pp.42).

\section{Los esfuerzos más recientes en Chile}

En el contexto chileno; el Ministerio de Vivienda y Urbanismo (MINVU) ha constituido comisiones de trabajo y realizado estudios que apuntan al reenfoque conceptual de los sistemas de planificación de asentamientos humanos poniendo de relieve a las personas como objeto y sujeto del desarrollo, enfatizando los objetivos en relación con la protección del medioambiente, a la necesidad de alcanzar mayor eficiencia en el uso del territorio y visualizando la gestión inmersa en el proceso de planificación y más específicamente en los instrumentos territoriales de planificación del uso del suelo. Sin embargo, para la operación de la ciudad, plantea (sólo al final de un documento oficial, de la serie Monografías y Ensayos, y en 3 páginas de 38), los elementos que a su juicio deben abordarse: eficiencia en la administración para alcanzar la gobernabilidad urbana $^{12}$, autofinanciamiento de la operación de la ciudad, descentralización de la gestión y participación ciudadana; pero no hay propuestas operacionales y se limita a indicar que: "No siempre hay conciencia de que la ciudad en un artefacto complejísimo que requiere ser administrado y que ello exige contar con potestades, recursos humanos, recursos financieros y organización". (Minvu, 1996; pp.36)

\footnotetext{
12 EI MINVU define Gobernabilidad Urbana como: identificar tareas a realizar y quien las hará, las funciones que deben descentralizarse y las que deben centralizarse. Véase en bibliografía MINVU, 1996, pp. 37 
También en Chile el Ministerio de Vivienda y Urbanismo, en el marco de la Consulta Nacional Para la Formulación de una Nueva Política de Desarrollo Urbano entre los años 1993-96 y con apoyo del Programa de Gestión Urbana de Naciones Unidas ${ }^{13}$, se desarrollaron esfuerzos que contribuyeron notablemente a la conceptualización y clarificación del rol de la administración y gestión urbanas como dos caras de una misma moneda entorno a la gobernabilidad de las ciudades. A pesar de que dichos esfuerzos no se han traducido en la implementación de una nueva política urbana oficial ${ }^{14} \mathrm{ni}$ tampoco en reformas sustantivas de la legislación urbana, se logró acumular contribuciones importantes como la de Galilea ${ }^{15}$ y Jordán (1996) con relación a la gobernabilidad urbana. La noción de gobernabilidad se refiere en general a la forma en que los gobiernos - y, más específicamente, las estructuras administrativas y las personas que ejercen cargos públicos a todo nivel - se vinculan y articulan con los gobernados, con los electores, con la ciudadanía. (op. cit.; pp. 308) Para el caso de los gobiernos urbanos, la gobernabilidad puede entenderse ... como una medida para la evaluación y desarrollo de propuestas destinadas a facilitar la acción de un gobierno. Ello, por supuesto, supone la existencia de un ente posible de ser gobernado (la ciudad); de una institución legitimada que gobierne (la municipalidad, la asociación de municipios, el consejo metropolitano, la alcaldía mayor, etc.); y la existencia de procedimientos y, en general, una estructura que vincule este gobierno con la ciudadanía o la base social, y con los otros niveles subnacionales y nacional de gobierno. (op. cit.; pp. 311).

En la tradición chilena, ... a diferencia de la sajona, el aparato de administración urbana forma parte de un continuo de autoridades de distintos niveles, organizados de forma jerárquica y con crecientes grados de discrecionalidad. Aunque la administración urbana ha venido fortaleciéndose, gracias a un sostenido proceso de descentralización administrativa y territorial, no logra aún constituirse en un gobierno urbano en los términos indicados. La administración urbana centra su atención en el corto plazo (en la provisión de servicios básicos, equipamientos, etc.); pero no en la promoción de estrategias de desarrollo y otros aspectos estratégicos de mediano y largo plazos. Por esta razón es que Galilea y Jordán indican que para el caso chileno, ... es de utilidad concebir la gobernabilidad como parte de un proceso de creación y fortalecimiento de gobiernos locales, y urbanos, por añadidura."(op. cit.; pp. 313).

Un aspecto muy relevante de la contribución de Galilea y Jordán es la noción del concepto de gestión como expresión concreta de la acción que se ve determinada por el marco que generan las condiciones de la gobernabilidad por cuanto esta última ... da cuenta de los estilos, instrumentos y, sobre todo, principios para la acción del gobierno urbano. En el trasfondo está la noción de gobierno, y con ella la de inducir, a través de las diferentes acciones gubernamentales, orientaciones al desarrollo, en este caso de la ciudad. Ello necesariamente incluye las dimensiones social, económica espacial-territorial y natural. (Op.cit). Pero la determinación de la gobernabilidad sobre la gestión se

\footnotetext{
13 Programa coordinado para Latinoamérica, hasta 1997, por el economista chileno Dr. Pablo Trivelli.

${ }^{14}$ Rigen hasta hoy, ante la carencia de una nueva y explícita política pública, los principios de la Política Urbana de 1985 diseñada en el Gobierno de Pinochet, recién derogada en octubre 2000.

${ }^{15}$ Sergio Galilea al momento del artículo era Subsecretario del MINVU, hasta el 2001 Intendente de la Región Metropolitana de Santiago, capital de Chile. (el Intendente es la máxima autoridad regional en Chile y la designa el Presidente de la República), y actualmente forma parte del equipo asesor político del Presidente R. Lagos. Revista de Urbanismo $N^{\circ} 7$ - Enero de 2003 
entiende en un contexto de relación, es decir como un par de conceptos que se asocian e interrelacionan y, por lo tanto, dicha determinación es mutua. "La gestión urbana encierra en sí un conglomerado de condiciones y atribuciones que se asocian a la gobernabilidad, estando ambas mutuamente determinadas". Ello implica que el fortalecimiento de la una requiere y lleva aparejado el de la otra. (Op. cit.; pp.314).

En definitiva, Galilea y Jordán definen que ... los propósitos de cualquier gobierno urbano hoy día, en respuesta a las condiciones de cambio político, institucional, económico y social, deberán incluir como tema frontera, (...): mejorar la productividad y la eficiencia urbana...". Es decir, la ciudad en tanto sistema, para servir de soporte eficiente a la producción y a un mejorado nivel de vida de la población, demanda de recursos de operación y de un sistema de administración también eficiente. (Op. cit.; pp. 316). Estos autores avanzan hacia una definición más acabada de la problemática indexando el tema de nuestro interés (administración y gestión urbana) al crucial asunto de la gobernabilidad urbana como mecanismo para fortalecer la capacidad de conducción de las ciudades en el contexto chileno. No hay, sin embargo aportes en el marco de los sistemas administrativos posibles, o de las distintas modalidades organizacionales para el gobierno urbano, o de modelos de gestión urbana.

Los esfuerzos más recientes están destinados a concretar una Reforma Urbana ${ }^{16}$ cuyo perfil fue definido por el Presidente Lagos, en su cuenta pública al país desde el Parlamento en mayo de 2001, en los siguientes términos: Se hace urgente transformar en una nueva política el rol central que las ciudades juegan en la generación de oportunidades para las personas y en el desarrollo del país, introduciendo cambios estructurales a los procesos y formas de desarrollo urbano, descentralizando los esfuerzos públicos, ampliando los grados de autonomía e iniciativa urbana a las comunidades locales, flexibilizando la operación de los mercados urbanos y abriendo las ciudades a la innovación, la creatividad y la participación ${ }^{17}$.

\section{Modernización de la administración y gestión urbana}

Los problemas y el rol de las administraciones urbanas tienden a tocarse en el contexto de la planificación estratégica vinculada al proceso de orientación y regulación de las ciudades. En efecto, los esfuerzos de España en el marco europeo, específicamente los casos de Barcelona, Valencia y Bilbao; se muestran como paradigmáticos para la planificación. En esos casos se destaca el rol central de la administración y gestión urbanas y la necesidad de invertir recursos y esfuerzos en pos de su modernización. Se muestra, además, la impresionante incapacidad y resistencia de la administración para ponerse acorde con la alta velocidad de cambio de su entorno especialmente en los últimos años. Estas experiencias permiten visualizar la necesidad de instituciones flexibles y adaptables ${ }^{18}$.

\footnotetext{
${ }^{16}$ Al respecto véanse en bibliografía:

MINVU 1, Bases para la Reforma de la Ciudad y el Territorio, Documento $N^{\circ} 1$, marzo, 2001.

MINVU 2, Plan de Reforma Urbana y Territorial, Documento Interno, abril, 2001.

17 Discurso de S.E. el Presidente de la República, D. Ricardo Lagos Escobar, en la Cuenta Anual al País, Valparaíso, 21 de mayo de 2001.

${ }^{18}$ Para más detalles del caso español véase en bibliografía Fernández, 1997.

Revista de Urbanismo $N^{\circ} 7$ - Enero de 2003 
Al respecto son atingentes las reflexiones de Osborne y Gaebler en relación con la sociedad norteamericana: Pero el modelo burocrático se desarrolló en condiciones muy diferentes de las que hoy en día debemos enfrentar. Se desarrolló en una sociedad de ritmo lento, en que los cambios tenían lugar pausadamente. Se desarrolló en la época de la jerarquía, cuando sólo los que se hallaban en la cumbre de la pirámide disponían de información suficiente como para adoptar decisiones con fundamento. Se desarrolló en una sociedad de gente que trabajaba con las manos, no con la mente. Se desarrolló en una época de mercados masivos, en que la mayoría de los norteamericanos tenían deseos y necesidades parecidas. Se desarrollo, por último, cuando teníamos vigorosas comunidades geográficas: vecindades y ciudades firmemente entretejidas. Hoy en día todo eso ha desaparecido. Vivimos en una asombrosa época de cambio. Vivimos en un mercado global que ejerce una enorme presión competitiva sobre nuestras instituciones económicas. Vivimos en una sociedad de información, a la cual la gente accede casi tan rápidamente como sus lideres. Vivimos en una economía basada en el conocimiento, en la cual los trabajadores con formación manejan los mandos y exigen autonomía. Vivimos en una época de unos mercados en los que los consumidores se han acostumbrado a la gran calidad y amplitud de las opciones posibles.

En este medio, instituciones burocráticas- públicas y privadas - que se desarrollaron durante la era industrial nos resultan cada vez más inútiles.

El medio actual exige instituciones extremadamente flexibles y adaptables. Exige instituciones que ofrezcan bienes y servicios de gran calidad y que aprovechen al máximo todas las energías. Exige instituciones responsables ante sus clientes, que brinden servicios no estandarizados; que gobiernen más por la persuasión y los incentivos que mediante órdenes; que creen en sus empleados un sentimiento de significado, de control e incluso de propiedad." (D. Osborne; T. Gaebler, 1994, pp. 43)

El asunto de la mayor velocidad de cambio del entorno que del aparato de administración y gestión del urbanismo forma parte, sin duda del contexto mayor que presiona la modernización del Estado en el marco que, a su vez, predefinen los vertiginosos avances tecnológicos a escala planetaria. En Chile, el sector privado en la construcción (que pasó desde un $23 \%$ de participación en el total edificado en 1980 a un $81 \%$ en $1997^{19}$ ), actuando en un contexto de mercado abierto al mundo, libre y altamente competitivo, se ve sometido permanentemente a la necesidad de innovar y rediseñar en productos y procesos no sólo en el área de la producción sino también y, especialmente, en la administración y gestión porque su supervivencia como empresa depende de aquello.

En la misma dirección y contexto, los organismos gubernamentales se constituyen en un factor crítico tanto para la promoción como para la regulación y control en tanto marco de los demás actores del proceso de producción de bienes y servicios urbanos; y están siendo fuertemente presionados por el sector privado/comunitario para que el comportamiento de la administración no se constituya en un obstáculo para su desempeño. Pero la presión sobre la administración también viene desde el sector comunitario ciudadano $\mathrm{y}$, en este caso, con miras a obtener más, mejores y más

\footnotetext{
${ }^{19}$ Véase MI NVU 1, 2001.

Revista de Urbanismo $\mathrm{N}^{\circ} 7$ - Enero de 2003
} 
eficientes servicios públicos. De hecho, una administración eficiente puede marcar la diferencia en el proceso de toma de decisiones del sector privado/comunitario para la instalación de sus actividades en un territorio u otro. Por ejemplo en Chile, en el Area Intercomunal de Concepción, una de las ciudades (Coronel) logró cambiar su rol minero tradicional hacia uno industrial-pesquero a partir de la mayor competitividad ganada por sobre la ciudad competidora más cercana. Dicha ventaja se obtuvo por un fuerte incremento de la eficiencia de su administración y gestión urbanas, medida esta en: menores tiempos en el proceso de aprobación de permisos de construcción, promoción sobre la base del aporte de terrenos para incentivar la instalación de industrias, rebajas de impuestos, etc. Es necesario insistir sin embargo, que cuando los esfuerzos son aislados y no implican el rediseño de procesos sino más bien son producto de la fuerte voluntad política unipersonal de la autoridad circunstancial, no se institucionalizan y, por lo tanto, contienen un alto riesgo de desaparecer porque el estilo de gestión tiende a seguir la misma suerte del líder.

En general, el desfase de la modernización entre las administraciones y la empresa privada tiende a explicarse por la falta de recursos financieros, sin embargo, tal como lo indica Gates (1999) "La razón de este desfase no es tanto la falta de dinero como una falta de enfoque organizativo" (op. cit.; pp. 403). Los servicios que presta la administración urbana constituyen, por definición en la legislación chilena y mundial, un monopolio y, en el caso de las áreas metropolitanas, un oligopolio pero con escasa movilidad de elección por parte del usuario; y entonces, “... las entidades de la administración acaban fijándose exclusivamente en las necesidades internas de su propia organización y en el cumplimiento a la letra de los reglamentos, no en atender las necesidades en general de los ciudadanos y de las empresas". (...) “...Para el ciudadano corriente o la empresa media la administración sigue siendo un enredo intimidante de entidades y reglamentaciones mal coordinadas" (Gates, op. cit; pp.404). Como propuesta gruesa de solución, el líder de la Microsoft plantea la digitalización de los procesos, “... los procesos digitales y el estilo de vida en la red ofrecen a las autoridades la oportunidad de reinventarse a sí mismas orientándose más de cara a la ciudadanía y menos a la burocracia". (op. cit.)

Del planteamiento anterior podría estimarse que la digitalización de los procesos soluciona los procesos en sí mismo; pero resulta obvio que tal como Gates visualiza que el problema de la ineficiencia de las administraciones no está principalmente en la falta de dinero (aunque este es necesario), nos parece que la dificultad no está en la digitalización de procesos existentes sino en el desenfoque organizativo que el mismo Gates planteaba en principio. La incorporación de las tecnologías de información ( $\mathrm{TI}$ ), tal como el dinero, resultan ser condiciones necesarias aunque no suficientes para enfrentar el proceso de modernización de la administración y gestión del urbanismo. Sin embargo, en el caso de las TI, el gran marco de posibilidades que abre por la síntesis de conocimiento incorporado; por las grandes facilidades que ofrece a la coordinación de procedimientos, métodos y formularios; por las altas velocidades disponibles para la transmisión de datos; por las economías de escala que hacen factible de alcanzar relativamente bajos costos de inicio; presentan oportunidades interesantes al rediseño de procesos para agilizarlos, coordinarlos, con miras a un servicio a las personas y empresas más eficiente, confiable y a menor costo. 
Gestión del urbanismo y administración urbana: Los pies de barro en el despliegue territorial del neoliberalismo en Chile

\section{El Caso de Singapur: rediseñar la administración para ganar competitividad}

Con relación a lo anterior, el caso de la ciudad-estado de Singapur ${ }^{20}$, que con sus 2,6 millones de habitantes viviendo en una isla de $625 \mathrm{~km}^{2}$, muestra quizás la mayor evidencia mundial de un rediseño exitoso de procesos apoyados por TI. Las autoridades y la administración, trabajando de conjunto con el sector privado, llegaron a la conclusión de que debían hacer más eficiente el comercio. Esto significaba construir soporte tecnológico; pero quizás más importante, coordinar los procesos y protocolos de muchas agencias y organizaciones diferentes en un set de procedimientos simples y coherentes que pudieran ser automatizados. Abordaron las tareas de rediseñar procesos por cuanto "automatizar procesos existentes produciría nada más que automatizar un caos". (Harvard; 1995; pp.5) Así nació el TradeNet, un sistema electrónico que permite el intercambio de documentos entre empresas en el marco de la comunidad de comercio de Singapur y que permitió que la ciudad mantuviese el liderazgo en el comercio de intercambio portuario en relación con su competidora más cercana: la ciudad de Hong Kong.

El Trade Net constituyó entonces un modelo de administración y gestión de la actividad comercial portuaria en Singapur facilitando el marco y estimulando la producción de bienes y servicios. Se apuntó allí a solucionar el problema central: la burocracia como factor inhibidor de la eficiencia en el desempeño del rol principal de una ciudad.

\section{El Caso de Curitiba: liderazgo y asociatividad}

Otro caso relevante de fortalecimiento de la gestión como mecanismo para avanzar en la solución de los problemas urbanos es el de la ciudad de Curitiba, Estado de Paraná en el sur de Brasil. A partir de 1971 fue elegido Alcalde de la ciudad el arquitecto Jaime Lerner (y posteriormente Gobernador del Estado). La ciudad con su millón y medio de habitantes, fue capaz de solucionar, mediante un sistema integrado de medios de transporte colectivo de superficie, sus graves problemas de congestión. Al respecto Lerner indica que: "esto fue una tarea que un equipo implantó durante todo el tiempo. Probó que no era una cuestión de recursos, que el problema era más una cuestión de gestión, de voluntad política de hacer el cambio". (Lerner, Gaete, et. alt.; 1997, pp. 46) Dice Lerner: Los problemas de (la ciudad) se pueden resolver de manera mucho más efectiva con el poder local. Yo quiero esclarecer un punto, Curitiba no es un paraíso; Curitiba tiene todos los problemas que las ciudades brasileñas tienen, que todas las regiones metropolitanas tienen; pero, ¿cuál es la diferencia?. La diferencia está en el respeto, esto significa respeto al ciudadano. Cuando el ciudadano se siente respetado asume una corresponsabilidad para con su ciudad y con su país (op.cit.; pp. 49). Para avanzar en la solución de los problemas urbanos, dice Lerner, es necesario primero ... una visión estratégica, porque no se hace cambio sin visión estratégica." Pero es interesante como el brasileño lleva el asunto a la administración y gestión cotidiana, al

\footnotetext{
${ }^{20}$ Para una revisión exhaustiva del caso, véase en bibliografía, Harvard Business School, 1995.
} 
decir: "En mi opinión la visión estratégica se da con un balance diario de necesidades y potencialidades. Si una ciudad se ocupa solamente de las necesidades vamos siempre a estar corriendo tras el problema, no habrá cambios. Pero si una ciudad se ocupa solamente de las potencialidades, un gran número de personas se va a ir del pueblo. Entonces cuando no hay recursos, y nunca hay recursos, en cualquier ciudad del mundo la solución es encontrar una ecuación de corresponsabilidad. ... no hay problema urbano que no se pueda resolver con una ecuación de corresponsabilidad... (op. cit.; pp. 50). Y ante la pregunta ¿cuál es el secreto de Curitiba?, Lerner responde; “...el primer secreto es simplicidad, no tener miedo de hacer soluciones simples..." (op. cit.; pp. 52) Y agrega: Resolver una ciudad es resolver un sueño colectivo. Cambiar una ciudad es cambiar un sueño colectivo. (...) Para intentar resolver un sueño colectivo tenemos que encontrar una causa compartida, si la ciudad no se transforma en una causa compartida no se va a cambiar (op. cit.; pp 53).

El caso de Curitiba, para el contexto latinoamericano, se nos presenta como una experiencia paradigmática en tanto modelo de gestión a partir de un marcado liderazgo de su Alcalde, con alta capacidad para formar equipos técnicos con visión compartida en torno a un proyecto; pero también con clara definición para colocar en el centro de las preocupaciones al ciudadano y su vida cotidiana. En esa dimensión se juegan los procesos con fórmulas y procedimientos simples para facilitar la integración al esfuerzo colectivo (corresponsabilidad) Este es entonces, un modelo que apuntó allí a solucionar el problema central de falta de recursos por la vía del incentivo a la asociatividad.

\section{Conclusiones y proposiciones}

La gestión del urbanismo y la administración urbana forman parte en Chile de la esfera de acción de la Administración Pública y, por lo tanto, son herramientas del Estado para asegurar el bien común en el territorio, resultando ser claves para su orientación y regulación.

El abordaje eficiente de los problemas urbanos en el Chile de hoy requiere enfrentar asuntos centrales específicos que han quedado rezagados o fuera de los llamados procesos de modernización llevados adelante en el marco del modelo económico neoliberal vigente: de una parte, la ineficiencia en la administración y gestión del desarrollo territorial $y$, de otra, la insuficiencia de financiamiento para el mismo. En tanto constituyen lastres relevantes del sistema, eliminarlos agilizaría la gestión con el consiguiente aporte al fortalecimiento del dinamismo en las actividades urbanas. A nuestro juicio, abordar el primer asunto en forma integral incluye al segundo porque, en su seno, se debe incorporar el instrumental y los mecanismos de financiamiento necesarios.

Especialmente en regiones, es crucial el fortalecimiento de la capacidad técnica de los órganos responsables del desarrollo urbano dotándolos de los recursos humanos, métodos e instrumental adecuados para abordar, con mayor racionalidad, las acciones orientadas a posibilitar un desarrollo que satisfaga las necesidades de los habitantes y de la propia actividad económica y de producción. Estos órganos gubernamentales deberían estructurarse, a bajo costo recurrente y para fortalecer sus grados de asociatividad, 
constituyendo, simultáneamente, una pequeña; pero ágil, Red Física y una grande, eficiente y poderosa Red Virtual apoyada en tecnologías de información.

El estado del arte en los temas que nos preocupan, muestra importantes oportunidades de aporte al conocimiento en la modelación para la operación de la administración y gestión urbanas, con sus instrumentos, mecanismos, sistemas de información, articulación de actores, indicadores para la evaluación, etc.; a través de propuestas que apunten al diseño y/o rediseño de los procesos aprovechando las posibilidades que abren, por ejemplo, las tecnologías de información en pos de fortalecer la capacidad de respuesta de los organismos responsables de orientar, regular, administrar y gestionar la ciudad. En esta dirección la alianza estratégica entre los Gobiernos Regionales y Locales con el aparato universitario viabilizaría soluciones en el marco de un Programa de Modernización.

El diseño de modelos de gestión y administración urbanas debe estar acorde a los desafíos de integración de la población, a los beneficios del desarrollo, y a la articulación de los sectores público, y privado/comunitario, asuntos que se constituyen en una necesidad crucial para el desarrollo de Chile. En el primer caso nos referimos a que la concepción del modelo debe apuntar a alcanzar un objetivo ético-político ya que se trata de priorizar el estilo de desarrollo territorial y urbano como una palanca para asegurar equidad e igualdad de oportunidades en el acceso a niveles adecuados de habitabilidad en el territorio para todos los ciudadanos. En el segundo caso estimamos que el sector privado/comunitario debería articularse con el público aportando, además del tradicional papel de desarrollador y materializador de proyectos de los agentes del área privada/privada, hacia un rol más activo en el ejercicio de funciones públicas delegadas. Por ejemplo en: sistemas de información internos, evaluación de planes, programas y proyectos, servicios integrales al usuario, organización, educación y participación ciudadana.

Diciembre, 2002.

\section{Referencias}

A.- Documentos y Textos Oficiales de Órganos Gubernamentales de la República de Chile.

1. REPÚBLICA DE CHILE, Constitución Política del Estado, Gobierno de Chile, 1980-1996.

2. LAGOS, R, Discurso de S.E. el Presidente de la República, D. Ricardo Lagos Escobar, en la Cuenta Anual al País, Valparaíso, 21 de mayo de 2001.

3. LAGOS, R, Intervención de S.E. el Presidente de la República, D. Ricardo Lagos Escobar, en ceremonia de anuncio de la Reforma Urbana, Santiago, 25 de junio de 2001.

4. MINVU 1, Bases para la Reforma de la Ciudad y el Territorio, Documento N¹, marzo, 2001.

5. MINVU 2, Plan de Reforma Urbana y Territorial, Documento Interno, abril, 2001.

6. MINVU 3, Política Nacional de Desarrollo Urbano, Decreto Supremo (D.S.) №31/1985, MINVU, 4/3/1985. (derogado en octubre de 2000 por decreto MINVU N²59)

7. MINVU 4, Ley General de Urbanismo y Construcciones, Decreto con Fuerza de Ley (D.F.L.) № 458/ 1975, Ministerio de Vivienda y Urbanismo, (MINVU), Diario Oficial, (D.O.) 13/4/1976.

8. MINVU 7, SILVA, Jaime, y otros, Desarrollo Urbano y Territorial, Santiago, Chile, 1996.

9. MINVU/PGU/UN 9, Chile Urbano. Antecedentes de la Consulta Nacional para la Formulación de una nueva Política de Desarrollo Urbano. Volumen 7, Programa de Gestión Urbana de las Naciones Unidas (PGU/NU). Oficina Regional para América Latina y el Caribe. 1996. 
10. MINTERIOR, Ley Orgánica Constitucional de Municipalidades, Decreto Supremo (D.S.) № 662/1992, Ministerio del Interior, D.O. 27/8/1992. (Texto refundido Ley 18.695), 1988.

11. MINTERIOR, Ley de Bases Generales del Medioambiente, N¹9.300, Diario Oficial, Chile, 09/Marzo/1994.

B.- Textos y Documentos Gubernamentales Oficiales de Otros Organismos.

12. BANCO MUNDIAL - UNCHS - HABITAT - PNUD, Programa de Administración Urbana, Fase 2: Fortalecimiento de la Administración Urbana en el Decenio, 1990.

13. CEPAL/NU, La Crisis Urbana en América Latina y el Caribe, Reflexiones sobre Alternativas de Solución, Comisión Económica para A. L., (CEPAL), Naciones Unidas, Santiago, Chile, 1989.

14. UNCHS-HABITAT, What is a Sustainable City? . Nairobi, 1991.

15. N.U./HABITAT II, La Conferencia de las Naciones Unidas sobre los Asentamientos Humanos (Hábitat II), DECLARACIÓN DE ESTAMBUL SOBRE LOS ASENTAMIENTOS HUMANOS, PLAN DE ACCIÓN MUNDIAL: ESTRATEGIAS PARA LA APLICACIÓN, Estambul, junio, 1996.

16. UNITED NATIONS CENTRE FOR HABITAT, Implementing the Urban Environment Agenda. Volumen 1. 1997.

C.- Textos y Documentos de Otros Autores.

17. BOISIER, Sergio, Regionalización, Descentralización y Desarrollo Regional, CEPAL, ILPES, 1990.

18. BOISIER, Sergio, La Gestión Regional, Serie Ensayos, ILPES/ ONU. Santiago, Chile, 1991.

19. BOISIER, Sergio, Notas Sobre Regionalización, Descentralización y desarrollo Regional, Serie Ensayos, ILPES/ ONU. Santiago, Chile, 1990.

20. CASTELLS, M., BORJA, J., “Urbanización y Crisis Urbana en América Latina”, en La Ciudad de la Democracia, varios autores, Centro de Estudios Económicos y Sociales VECTOR, Ediciones Documentas, Santiago, Chile, 1988, pp. 9-30.

21. CORCHILE/CORBIOBIO, Gestión Política y Desarrollo Armónico de Chile. VI Jornadas nacionales de regionalización, CORBIOBIO, Concepción, 1990.

22. CONAREDE, Todo Chile es Chile: Cumbre de las Regiones, (Consejo Nacional Para la Regionalización y el Desarrollo), Santiago, 1998.

23. FERNÁNDEZ, José Miguel, Planificación Estratégica de Ciudades, Editorial G. Gili, Barcelona, 1997.

24. FFRENCH-DAVIS, Ricardo, Entre el Neoliberalismo y el Crecimiento con Equidad. Tres Décadas de Política Económica en Chile, Editorial Dolmen, Santiago, Chile, 20001.

25. GAETE, Héctor, "Propiedad y Rol de los Sectores Público y Privado en el Desarrollo de la Ciudad", en libro DESARROLLO URBANO, Dpto. Planificación y Diseño Urbano, Universidad del Bío-Bío, Concepción, Chile, 1996, pp. 57-65.

26. GAETE, Héctor, “La Administración del Desarrollo Urbano en Chile. Marco Global para la Gestión Privada y Pública", en revista Theoria, Universidad del Bío-Bío, Volumen № 7, 1998.

27. GALILEA, Sergio., JORDAN, Pablo., “Gobernabilidad Urbana en Chile. Algunas Reflexiones, Enfoques y Propuestas", en CHILE URBANO: Antecedentes de la Consulta Nacional para la formulación de una Nueva Política de Desarrollo Urbano, PGU/MINVU, Santiago, Chile 1996, pp. 308 - 342.

28. HARVARD BUSINESS SCHOOL, Singapore Tradenet: A Tale of One City, Bower, M., Konsynski, B., Harvard, Cambridge, USA, 1995.

29. JOHANSEN, Oscar, El Administrador Como un Definidor, Facultad de Ciencias Económicas y Administrativas de la Universidad de Chile, Santiago, Chile 1998.

30. LERNER, Jaime., GAETE, Héctor., y otros, Las Ciudades en el Desarrollo Nacional. Desafíos para un Buen Gobierno, I. Municipalidad de Santiago, Chile, 1997.

31. OSBORNE, David, GAEBLER, Ted, La Reinvención del Gobierno. La Influencia del Espíritu Empresarial en el Sector Público, Ediciones Paidós I bérica, Barcelona, 1994

32. UBB/CEPAL, Instituciones y Actores del Desarrollo Territorial en el Marco de la Globalización, Ediciones Universidad del Bío-Bío, Concepción, Chile, 1999. 
Gestión del urbanismo y administración urbana: Los pies de barro en el despliegue territorial del neoliberalismo en Chile

33. VERGARA BLANCO, Alejandro, Marco Jurídico para un proceso de Descentralización Administrativa". CORBIOBIO, Concepción, Chile, junio, 1991.

34. RODRÍGUEZ, Alfredo y otros, Municipios Servicios Públicos, Gobierno Locales en Ciudades Intermedias de América Latina. Editorial Interamericana, 1994.

35. SMOLKA, Martín y otros, Recuperación de la Plusvalías en América. LOM Ediciones, Santiago, Chile, 2001.

36. ALVEZ CATALÁN, Hernán, Centralismo y Desarrollo Regional en Chile, Comunicadores Asociados Ltda., 1999.

37. BRUNNER, José J oaquín, Globalización Cultural y Posmodernidad, Editorial Siglo XXI, México, 1998.

38. ETCHEGARAY, Alberto, Huella y Visión de una gestión Ministerial, editado por Comunicaciones Despertar, Santiago, Chile, 1994.

39. GATES, Bill, Los Negocios en la Era Digital, Editorial Sudamericana, B.A, Argentina 1999, 527 p.

Nota: Héctor Guillermo Gaete Feres es Arquitecto, U. del Bío-Bío/Chile; Doctor en Gestión y Valoración Urbana (c), U. Politécnica de Cataluña/España; Magister en Urbanismo, U. De Chile; Diplomado en Gestión de Servicios Públicos y Urbanos, Beca OEA, CIDER, U de los Andes/Colombia; Diplomado en Uso Estratégico de Tecnologías de Información, U de Harvard, USA; Profesor Investigador, Director Boletín Mercado del Suelo Urbano en el Gran Concepción, Depto. Planificación y Diseño Urbano, U. del Bío-Bío/Chile; Consultor Naciones Unidas; Agencia de Cooperación Alemana (GTZ); Ministerio de Vivienda y Urbanismo y Ministerio de Obras Públicas/Chile. hgaete@ubiobio.cl 